\title{
Approximate Solutions for Certain Bidomain Problems in Electrocardiography
}

\author{
Peter R. Johnston \\ School of Biomolecular and Physical Sciences, \\ Griffith University, Nathan, Queensland, Australia, $4111^{*}$
}

(Dated: September 12, 2008)

\begin{abstract}
The simulation of problems in electrocardiography using the bidomain model for cardiac tissue often creates issues with satisfaction of the boundary conditions required to obtain a solution. Recent studies have proposed approximate methods for solving such problems by satisfying the boundary conditions only approximately. This paper presents an analysis of their approximations using a similar method, but one which ensures that the boundary conditions are satisfied during the whole solution process. Also considered are additional functional forms, used in the approximate solutions, which are more appropriate to specific boundary conditions.

The analysis shows that the approximations introduced by Patel and Roth generally give accurate results. However, there are certain situations where functional forms based on the geometry of the problem under consideration can give improved approximations. It is also demonstrated that the recent methods are equivalent to different approaches to solving the same problems introduced twenty years earlier.
\end{abstract}

PACS numbers: $87.85 \mathrm{Tu}, 87.19 \mathrm{Hh}$

\section{INTRODUCTION}

Studying the electrical behaviour of heart tissue can provide insights into the way the electrocardiogram (ECG) (used as a common diagnostic tool by medical practitioners) works. These studies can also explain abnormalities in the ECG on the basis of abnormalities at the cellular level. One such application is the assessment of regions of heart tissue damage following a heart attack. Other applications of these studies are used to influence the design of cardiac pacemakers and defibrillators.

Cardiac tissue can be treated as a passive resistive medium in which Ohm's law governs the relationship between potential difference, tissue resistance and current flow. However, on a cellular scale conductivity inside a cell differs from conductivity outside the cell. Given that a typical cardiac cell is approximately $100 \mu \mathrm{m} \times 10 \mu \mathrm{m} \times 10 \mu \mathrm{m}$ [1], it would be computationally too expensive to study the electrical behaviour of heart tissue on the scale of the human heart. It has also been shown that cardiac tissue exists in sheets of fibres of cardiac cells [2], which also complicates the modelling.

The bidomain model [3] was introduced as a continuum approximation for the electrical properties of cardiac tissue which allows for the fibrous and cellular nature of the tissue. The model consists of an intracellular space (inside the cells) and an extracellular space (outside the cells, but within the cardiac tissue) and allows for anisotropic variations in electrical conductivity in both spaces along and across the direction of the fibres of cardiac cells. Ohm's law is assumed to apply in both of these domains which are further assumed to co-exist spatially.

Over recent years the bidomain model [3] has become the backbone of simulating many electrocardiographic phenomena. Examples of particular applications are the study of the electrocardiogram itself [4], defibrillation [5] and study-

*Electronic address: P. Johnston@griffith. edu . au ing subendocardial ischaemia in cardiac tissue [6-10]. Solutions to the bidomain equations have been achieved via numerical methods [10-13], combined analytical and numerical methods [6-9, 14, 15] and approximate analytical methods [4, 5, 16-18].

A conundrum which arises in the formulation of the bidomain model is: what are the correct boundary conditions to apply at the interface between the tissue and the surrounding medium (the so-called tissue-bath interface)? Generally, the extracellular tissue potential and the bath potential must satisfy three boundary conditions at the interface, as described in the next section. Through an insightful series of papers, Roth and various co-workers $[4,5,16,17]$ have devised a simple approximation to account for this conundrum and achieve approximate analytical solutions to the bidomain equations. The idea is to simply introduce an exponentially small quantity in the expressions for the potentials, solve for the potentials and then let the coefficients of the exponential terms tend to zero. This method produces very accurate solutions to the bidomain equations. However, the one small drawback with this idea is that the introduced exponential functions do not necessarily satisfy the boundary conditions on other boundaries in the problem.

In this paper, a modified approximation method, based on introducing, what will be called auxiliary functions, which satisfy all boundary conditions, is presented. In other words, the functions are based on the geometry of the problem under consideration. It is shown that for defibrillation problems this new approximation can be an improvement over previous approximations and can even yield exact solutions.

For cardiac propagation problems the approximations introduced by Patel and Roth [4] are quantified using a method based on choosing auxiliary functions which satisfy the boundary conditions combined with making fewer assumptions during the solution process. A detailed analysis of the approximations shows that the approximate solutions obtained by Patel and Roth are in general equivalent to those obtained by Roth and Wikswo [19] based on a different set of approximating assumptions. Finally, a slightly different 
formulation is introduced to model subendocardial ischaemia and the solutions obtained are compared with previously published solutions [6] and with full numerical solutions.

\section{GENERAL METHOD}

The bidomain equations which govern the intra- and extracellular potentials in cardiac tissue, $\phi_{i}$ and $\phi_{e}$, respectively, are

$$
\nabla \cdot\left(\mathbf{M}_{i} \nabla \phi_{i}\right)=I_{m}
$$

and

$$
\nabla \cdot\left(\mathbf{M}_{e} \nabla \phi_{e}\right)=-I_{m}
$$

where $\mathbf{M}_{i}$ and $\mathbf{M}_{e}$ are the intracellular $(i)$ and extracellular (e) conductivity tensors, respectively, and $I_{m}$ is the membrane current. Equations (1) and (2) are essentially statements of Ohm's law in the intracellular and extracellular spaces, respectively. The tensors $\mathbf{M}_{i}$ and $\mathbf{M}_{e}$ (represented via matrices) reflect the fact that the electrical conductivity of the cardiac tissue is anisotropic, since it is much easier for current to flow along the fibres than across them. Four conductivities are required to describe the behaviour of the tissue: intracellular $(i)$ and extracellular $(e)$ conductivities both along the fibres (longitudinal $(l)$ ) and across the fibres (transverse $(t)$ ). These conductivities are denoted by $\sigma_{i}^{l}, \sigma_{e}^{l}, \sigma_{i}^{t}$ and $\sigma_{e}^{t}$. Note that six conductivities are not required as it is assumed that the conductivities in both transverse directions are equal. Further, if the fibres rotate while moving through the tissue, the tensors also depend on the local fibre directions.

Through the membrane current, $I_{m}$, the bidomain equations (1) and (2) are coupled, but following Patel and Roth [4], they can be decoupled by defining the transformations

$$
\phi_{m}=\phi_{i}-\phi_{e} \text { and } \psi=\frac{\alpha}{1+\alpha}\left(\phi_{i}+\frac{1}{\alpha} \phi_{e}\right)
$$

with inverse transformations

$$
\phi_{i}=\psi+\frac{1}{1+\alpha} \phi_{m} \text { and } \phi_{e}=\psi-\frac{\alpha}{1+\alpha} \phi_{m} .
$$

In these transformations $\phi_{m}$ is the transmembrane potential, $\psi$ is an auxiliary potential and $\alpha$ is an as yet unspecified constant. Using these new potentials, and adding equations (1) and (2), it turns out that

$$
\nabla \cdot\left(\mathbf{M}_{i}+\mathbf{M}_{e}\right) \nabla \psi=-\frac{1}{1+\alpha} \nabla \cdot\left(\mathbf{M}_{i}-\alpha \mathbf{M}_{e}\right) \nabla \phi_{m} .
$$

For some applications of the bidomain equations it is assumed that $\phi_{m}$ (equation (3)) can be specified; in which case, adding equations (1) and (2) leads to

$$
\nabla \cdot\left(\mathbf{M}_{i}+\mathbf{M}_{e}\right) \nabla \phi_{e}=-\nabla \cdot \mathbf{M}_{i} \nabla \phi_{m},
$$

where neither the auxiliary potential $\psi$ nor the unspecified constant $\alpha$ are required and the extracellular potential can be obtained directly. In the context of this paper, either equation (5) or equation (6) will be solved, depending on the example under consideration.

Finally, to complete the formulation of the problem, assume that the tissue is surrounded by a bath of conductivity $\sigma_{b}$, where the bath potential $\phi_{b}$ satisfies Laplace's equation

$$
\nabla^{2} \phi_{b}=0
$$

To solve the problem a set of boundary conditions is required. Usually, most of the boundary conditions which depend on the geometry of the particular problem under consideration, are at the boundaries of the simulation domain, but boundary conditions at the tissue-bath interface are common to all problems:

1. The extracellular potential is equal to the bath potential

$$
\phi_{e}=\phi_{b}
$$

2. The normal component of the extracellular current density is equal to the normal component of the bath current density

$$
\sigma_{e}^{t} \frac{\partial \phi_{e}}{\partial n}=\sigma_{b} \frac{\partial \phi_{b}}{\partial n}
$$

3. The normal component of the intracellular current density is zero

$$
\sigma_{i}^{t} \frac{\partial \phi_{i}}{\partial n}=0
$$

where $n$ is the direction perpendicular to the surface, going into the tissue. In terms of the new potentials $\phi_{m}$ and $\psi$ these boundary conditions become

$$
\begin{aligned}
\psi-\frac{\alpha}{1+\alpha} \phi_{m} & =\phi_{b} \\
\sigma_{e}^{t}(1+\alpha) \frac{\partial \psi}{\partial n} & =\sigma_{b} \frac{\partial \phi_{b}}{\partial n} \\
\frac{\partial \phi_{m}}{\partial n} & =-(1+\alpha) \frac{\partial \psi}{\partial n}
\end{aligned}
$$

Alternatively, in the formulation based on $\phi_{m}$ and $\phi_{e}$ (equation (6)), the boundary conditions (8) and (9) remain the same and the boundary condition (10) becomes

$$
\frac{\partial \phi_{m}}{\partial n}=-\frac{\partial \phi_{e}}{\partial n}
$$

As highlighted by Patel and Roth [4, 5], making various assumptions about the form of $\phi_{m}$ does not guarantee satisfaction of all three boundary conditions. To overcome this problem for defibrillation problems [5], the authors initiate an iteration scheme by setting

$$
\phi_{m}=A e^{-n / \lambda}
$$

where $A$ is a yet to be determined function, independent of the normal direction $n$. The form $A e^{-n / \lambda}$ is used solely to 
satisfy the boundary conditions at the bath-tissue interface. For cardiac propagation problems [4], the authors write

$$
\phi_{m}=\phi_{m}^{0}+A e^{-n / \lambda}
$$

where $\phi_{m}^{0}$ presents a planar wave front which is a specific source term and $\lambda$ can be taken to be the space constant in the transverse direction. In order to allow for this additional term and to solve the differential equations the authors [4] also write

$$
\psi=\psi_{0}+B e^{-n / \lambda}
$$

In the context of this paper, the exponential function used in (15), (16) and (17) will be referred to as an auxiliary function. In what follows other functional forms of the auxiliary function will be considered.

The choice of the exponential function, as an auxiliary function, is a perfectly logical choice for a tissue of semiinfinite extent in the $n$ direction. In this instance, the exponential function decays to zero as $n$ increases and will decay rapidly when $\lambda$ is small. However, if the tissue is of finite extent in the $n$ direction then the exponential function may not satisfy other boundary conditions required by the physical situation and another form of the auxiliary function should be considered, typically hyperbolic functions or modified Bessel functions. Although these functions exhibit similar behaviours to the exponential function, they satisfy the necessary boundary conditions at other boundaries in the problem. The degree to which these functions mimic the behaviour of exponential functions depends on the parameter $\lambda$, so if the scale over which the problem is defined is large compared to $\lambda$, choosing an auxiliary function to be a simple exponential function is perfectly adequate. However, if the scale is of the order of $\lambda$ or smaller, then a more appropriate auxiliary function should be chosen.

The best way to highlight this new approach is via a number of examples.

\section{EXAMPLE 1: CYLINDRICAL STRAND IN A UNIFORM ELECTRIC FIELD}

Consider a cylindrical strand of cardiac tissue of radius $a$ in an electric field $E_{0}$ perpendicular to the strand (Figure 1). It is assumed that the fibres lie along the strand (into the plane of Figure 1) and that end effects can be ignored, meaning that the potentials are independent of $z$. Further, assume that the intracellular and extracellular conductivities in the transverse directions are equal, that is, $\sigma_{i}^{x}=\sigma_{i}^{y}=\sigma_{i}^{t}$ and $\sigma_{e}^{x}=\sigma_{e}^{y}=$ $\sigma_{e}^{t}$. Due to these assumptions, the zeroth term in Patel and Roth's iteration scheme [5] is the full solution to the problem. Hence, the governing equations for $\psi$ and $\phi_{b}$ in a cylindrical coordinate system are

$$
\frac{1}{r} \frac{\partial}{\partial r}\left(r \frac{\partial \psi}{\partial r}\right)+\frac{1}{r^{2}} \frac{\partial^{2} \psi}{\partial \theta^{2}}=0
$$

and

$$
\frac{1}{r} \frac{\partial}{\partial r}\left(r \frac{\partial \phi_{b}}{\partial r}\right)+\frac{1}{r^{2}} \frac{\partial^{2} \phi_{b}}{\partial \theta^{2}}=0
$$

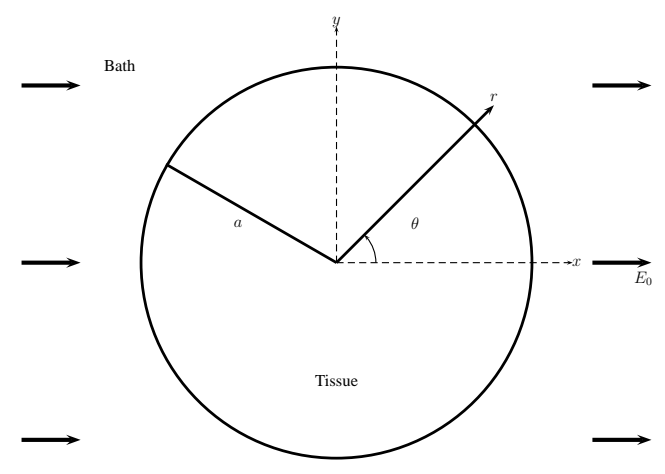

FIG. 1: Defibrillation of a strand of cardiac tissue. A cylindrical strand of radius $a$ placed in a bath with a uniform electric field of strength $E_{0}$ applied perpendicular to the strand.

subject to the boundary conditions (11), (12) and (13). At large values of $r$, the electric field is uniform in the $x$ direction, so $\phi_{b}$ becomes $-E_{0} r \cos \theta$. Therefore, the general solutions to (18) and (19) can be written as

$$
\begin{aligned}
\psi & =B r \cos \theta \\
\phi_{b} & =-E_{0} r \cos \theta+\frac{C}{r} \cos \theta
\end{aligned}
$$

where $B$ and $C$ are unknown constants.

Patel and Roth [5] suggest that the transmembrane potential, $\phi_{m}$, should fall exponentially with depth below the surface. However, from a physical stand point, it is also required that $\phi_{m}=0$ at $r=0$, so a better choice for the auxiliary function would involve modified Bessel functions. Hence, the form of $\phi_{m}$ is chosen to be

$$
\phi_{m}=A \cos \theta \frac{I_{1}(r / \lambda)}{I_{1}(a / \lambda)}
$$

where $I_{1}$ is the first kind modified Bessel function of order 1 . This choice of auxiliary function exhibits exponential decay with depth below the surface, takes the value $A \cos \theta$ at the surface and is zero when $r=0$. Substituting the approximation (20) into the boundary condition (13) gives

$$
A=-\left.(1+\alpha) \lambda \frac{I_{1}(a / \lambda)}{I_{1}^{\prime}(a / \lambda)} \frac{\partial \psi}{\partial r}\right|_{r=a}
$$

Further, substituting for $A$ from (21) in boundary condition (11) gives the new mixed boundary condition at $r=a$

$$
\phi_{b}=\psi+\lambda \alpha \frac{I_{1}(a / \lambda)}{I_{1}^{\prime}(a / \lambda)} \frac{\partial \psi}{\partial r}
$$

If it is assumed that $\psi=\phi_{b}$ at $r=a$ (following Patel and 


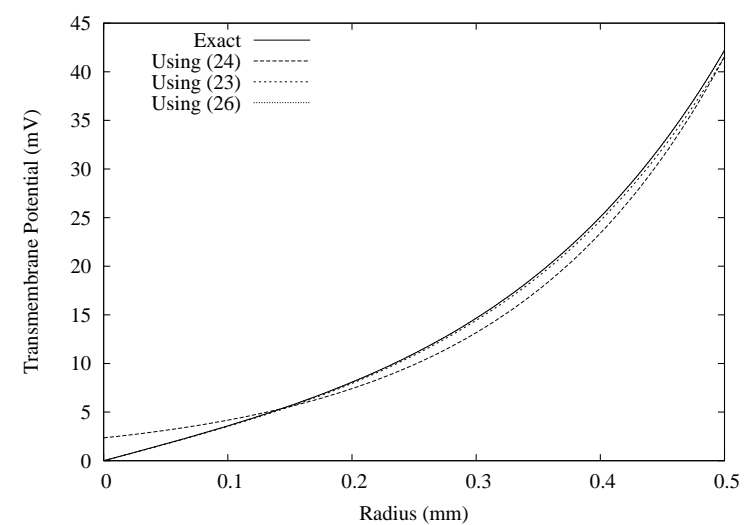

FIG. 2: Various approximate solutions and the exact solution for the transmembrane potentials plotted against radial distance for a cylindrical strand of cardiac tissue with $a=0.5 \mathrm{~mm}, \alpha=0.25$, $\lambda=0.174 \mathrm{~mm}, \sigma_{T}=0.0931 \mathrm{~S} / \mathrm{m}, \sigma_{b}=2 \mathrm{~S} / \mathrm{m}$ and $E_{0}=100 \mathrm{~V} / \mathrm{m}$.

Roth [5]), it follows that

$$
\begin{aligned}
& A=2 E_{0} \lambda(1+\alpha) \frac{I_{1}(a / \lambda)}{I_{1}^{\prime}(a / \lambda)} \frac{\sigma_{b} / \sigma_{T}}{1+\sigma_{b} / \sigma_{T}} \\
& B=-2 E_{0}\left(\frac{\sigma_{b} / \sigma_{T}}{1+\sigma_{b} / \sigma_{T}}\right) \\
& C=E_{0} a^{2}\left(\frac{1-\sigma_{b} / \sigma_{T}}{1+\sigma_{b} / \sigma_{T}}\right)
\end{aligned}
$$

giving

$$
\phi_{m}=2 E_{0} \lambda(1+\alpha) \frac{\sigma_{b} / \sigma_{T}}{1+\sigma_{b} / \sigma_{T}} \frac{I_{1}(r / \lambda)}{I_{1}^{\prime}(a / \lambda)} \cos \theta
$$

where $\alpha=\sigma_{i}^{t} / \sigma_{e}^{t}$ and $\sigma_{T}=\sigma_{i}^{t}+\sigma_{e}^{t}$.

Figure 2 shows a plot of the transmembrane potential $\phi_{m}$ (equation (23)), for a thin strand of cardiac tissue (of radius $0.5 \mathrm{~mm}$ ) along $\theta=0$, as well as a plot of the expression for $\phi_{m}$ obtained by Patel and Roth [5]

$$
\phi_{m}=2 E_{0} \lambda(1+\alpha) \frac{\sigma_{b} / \sigma_{T}}{1+\sigma_{b} / \sigma_{T}} e^{-(a-r) / \lambda} \cos \theta
$$

and the exact expression for $\phi_{m}[4]$

$$
\phi_{m}=2 a E_{0} \frac{1+\alpha}{\alpha} \frac{I_{1}(r / \lambda)}{I_{1}(a / \lambda)} \frac{1}{1+\frac{a}{\alpha \lambda}\left(1+\frac{\sigma_{T}}{\sigma_{b}}\right) \frac{I_{1}^{\prime}(a / \lambda)}{I_{1}(a / \lambda)}} \cos \theta .
$$

(Parameter values are given in the figure caption.) The figure shows that the new expression for $\phi_{m}$ (23) more closely matches the exact solution. In particular, there is a very good match for the inner $10 \%$ of the radius of the strand. This is opposed to the approximate solution (24) which shows its greatest deviation in this inner region. It is interesting to observe that both approximate solutions (23) and (24) tend to the same value at $r=a$, which is slightly lower than the exact value. This is presumably a result of the fact that both are trying to satisfy the approximate boundary condition $\psi=\phi_{b}$ at $r=a$.
The approximate solution (23) can be improved by actually applying the boundary condition (22). After some algebra it follows that

$$
A=2 a E_{0} \frac{1+\alpha}{\alpha} \frac{1}{1+\frac{a}{\alpha \lambda}\left(1+\frac{\sigma_{T}}{\sigma_{b}}\right) \frac{I_{1}^{\prime}(a / \lambda)}{I_{1}(a / \lambda)}}
$$

which, in turn, yields the exact solution to the problem. Hence, using an auxiliary function for the solution based on physical insights into the problem under consideration can yield an exact solution to the problem when no further approximations are made during the solution process.

\section{APPROXIMATIONS REVISITED}

Before considering an electrocardiographic type example with a propagation wavefront, it is worthwhile re-examining the approximations of Patel and Roth [4]. The idea with such problems is to solve the governing equations (5) and (7) subject to the boundary conditions (11), (12) and (13) with a specified $\phi_{m}$. As discussed above, Patel and Roth [4] introduce the forms (16) and (17) and substitute these into the equation (5) to obtain

$$
\nabla \cdot\left(\mathbf{M}_{i}+\mathbf{M}_{e}\right) \nabla \psi_{0}=0
$$

subject to the boundary conditions

$$
-\frac{\alpha}{1+\alpha} \phi_{m}^{0}+\psi_{0}=\phi_{b}
$$

and

$$
\sigma_{T} \frac{\partial \psi_{0}}{\partial n}=\sigma_{b} \frac{\partial \phi_{b}}{\partial n}
$$

at the bath-tissue interface.

These equations are derived under the assumption that propagation is along the fibre direction and that $\alpha=\sigma_{i}^{l} / \sigma_{e}^{l}$. It is also assumed that the coefficients $A$ and $B$ in equations (16) and (17) can be ignored since both $A$ and $B$ tend to zero as $\lambda \rightarrow 0$. Hence, in order to obtain approximate solutions to equations (1), (2) and (7) subject to the boundary conditions (8), (9) and (10) at the bath-tissue interface (as well as other geometry specific boundary conditions), one simply has to solve equations (27) and (7) subject to the boundary conditions (28) and (29) to yield $\psi_{0}$ and $\phi_{b}$.

Now examine these approximations in terms of the original functions $\phi_{i}$ and $\phi_{e}$. From equation (4) it follows that

$$
\phi_{i}=\psi+\frac{1}{1+\alpha} \phi_{m}=\psi_{0}+\frac{1}{1+\alpha} \phi_{m}^{0}
$$

since the coefficients $A$ and $B$ have been ignored. In turn, it also follows that

$$
\frac{\partial \phi_{i}}{\partial n}=\frac{\partial \psi_{0}}{\partial n}
$$

as $\phi_{m}^{0}$ is independent of depth in the $n$ direction. It further follows that $\frac{\partial \phi_{i}}{\partial n} \neq 0$ since equality is only obtained when the 
coefficients $A$ and $B$ are included, regardless of their magnitude. Further, by a similar argument it can also be shown that

$$
\frac{\partial \phi_{e}}{\partial n}=\frac{\partial \psi_{0}}{\partial n}
$$

With these approximations, consider the boundary condition (29):

$$
\begin{aligned}
\sigma_{b} \frac{\partial \phi_{b}}{\partial n} & =\sigma_{T} \frac{\partial \psi_{0}}{\partial n}=\left(\sigma_{i}^{t}+\sigma_{e}^{t}\right) \frac{\partial \psi_{0}}{\partial n} \\
& =\sigma_{i}^{t} \frac{\partial \psi_{0}}{\partial n}+\sigma_{e}^{t} \frac{\partial \psi_{0}}{\partial n}=\sigma_{i}^{t} \frac{\partial \phi_{i}}{\partial n}+\sigma_{e}^{t} \frac{\partial \phi_{e}}{\partial n}
\end{aligned}
$$

Hence, the approximate solutions $\phi_{0}$ and $\phi_{b}$ do not lead to solutions $\phi_{i}$ and $\phi_{e}$ which satisfy the boundary conditions (9) and (10), but to solutions which satisfy the weaker condition (33).

The above argument justifies the observation by Patel and Roth [4] that their approximate solutions obtained for propagation along a cylindrical fibre are equivalent to those derived by Roth and Wikswo [19] who assumed that $\phi_{m}$ was independent of depth and did not enforce the boundary condition (10), replacing the boundary condition (9) with the condition (33). Moreover, the above argument demonstrates that this observation is true for propagation in any geometry.

To summarise, in order to use the approximation method of Patel and Roth [4] and satisfy the boundary conditions (9) and (10) it is necessary to include the coefficients $A$ and $B$ in equations (16) and (17) both during the solution process and in the final solutions. Ignoring the coefficients $A$ and $B$ results in a solution which satisfies the boundary condition (33), which has been shown [20] to be an inappropriate boundary condition for use with the bidomain equations. Even though these coefficients have been shown to be very small in magnitude, their inclusion is necessary to solve the governing equations and boundary conditions correctly.

\section{EXAMPLE 2: TWO-DIMENSIONAL TISSUE ANALYSIS}

In light of the above discussion, the two-dimensional analysis of Patel and Roth [4] will be re-examined. Consider a slab of tissue in the region $z<0$, which is perfused by a bath $(z>0)$. Assume a planar wave front propagates in the $x$-direction (parallel to the fibres) with the wave front being independent of $y$ (Figure 3). Using the approximations (16) and (17) and previously mentioned assumptions, the governing equations are

$$
\frac{\partial^{2} \phi_{b}}{\partial x^{2}}+\frac{\partial^{2} \phi_{b}}{\partial z^{2}}=0
$$

and

$$
\sigma_{L} \frac{\partial^{2} \psi_{0}}{\partial x^{2}}+\sigma_{T} \frac{\partial^{2} \psi_{0}}{\partial z^{2}}=0
$$

where $\sigma_{L}=\sigma_{i}^{l}+\sigma_{e}^{l}$.

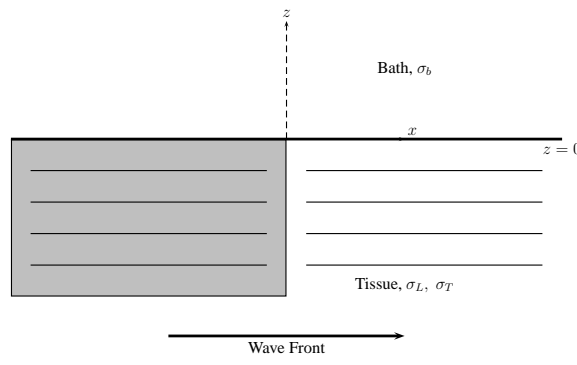

FIG. 3: A semi-infinite block of cardiac tissue, perfused by a bath of conductivity $\sigma_{b}$ containing a planar wave front propagating in the $x$-direction. The horizontal lines represent the fibre directions and the shaded area represents the action potential.

Patel and Roth [4] show that (in the notation of this paper)

$$
A=\left.\frac{\sigma_{e}^{t}+\sigma_{i}^{t}}{\sigma_{e}^{t}} \lambda \frac{\partial \psi_{0}}{\partial z}\right|_{z=0}
$$

and

$$
B=-\left.\frac{\sigma_{i}^{t}-\alpha \sigma_{e}^{t}}{(1+\alpha) \sigma_{e}^{t}} \lambda \frac{\partial \psi_{0}}{\partial z}\right|_{z=0}
$$

where $\alpha=\sigma_{i}^{l} / \sigma_{e}^{l}$.

Substituting the expressions (16) and (17) into the boundary conditions (11) and (12) gives the following boundary conditions at $z=0$ :

$$
\psi_{0}-\frac{\alpha}{1+\alpha} \phi_{m}^{0}-\lambda \frac{\sigma_{i}^{t}}{\sigma_{e}^{t}} \frac{\partial \psi_{0}}{\partial z}=\phi_{b}
$$

and

$$
\sigma_{T} \frac{\partial \psi_{0}}{\partial z}=\sigma_{b} \frac{\partial \phi_{b}}{\partial z}
$$

Assuming that $\phi_{m}^{0}$ varies sinusoidally in the $x$-direction with spatial frequency $k$ (i.e. $\phi_{m}^{0}=V_{0} \sin k x$ ), the solutions of (34) and (35) are then

$$
\phi_{b}(x, y)=C e^{-k z} \sin k x
$$

and

$$
\psi_{0}(x, z)=D e^{\delta k z} \sin k x
$$

where $\delta=\sqrt{\sigma_{L} / \sigma_{T}}$. Next, substituting (40) and (41) into the boundary conditions (38) and (39) gives

$$
C=-\frac{\alpha}{1+\alpha} V_{0} \frac{\sqrt{\sigma_{L} \sigma_{T}}}{\sigma_{b}} \frac{1}{1+\frac{\sqrt{\sigma_{L} \sigma_{T}}}{\sigma_{b}}+\lambda k \frac{\sigma_{i}^{l}}{\sigma_{e}^{i}} \sqrt{\frac{\sigma_{L}}{\sigma_{T}}}}
$$

and

$$
D=\frac{\alpha}{1+\alpha} V_{0} \frac{1}{1+\frac{\sqrt{\sigma_{L} \sigma_{T}}}{\sigma_{b}}+\lambda k \frac{\sigma_{i}^{l}}{\sigma_{e}^{i}} \sqrt{\frac{\sigma_{L}}{\sigma_{T}}}}
$$




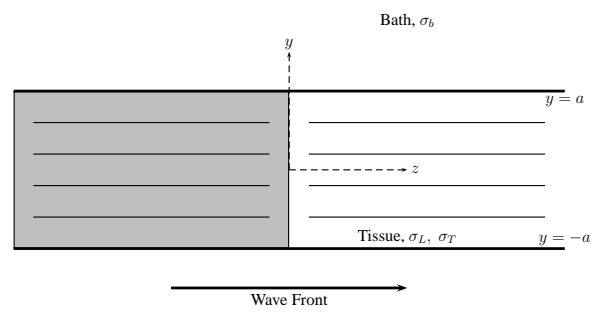

FIG. 4: A slab of cardiac tissue of thickness $2 a$, perfused by a bath of conductivity $\sigma_{b}$ on both sides (for $|y|>a$ ) with planar wave front propagating along the $z$-direction. The format is the same as Figure 3.

When compared with the respective coefficients obtained by Patel and Roth [4], both new coefficients contain a small correction factor which depends on the product $\lambda k$. The final solution for $\psi$ is then

$$
\begin{aligned}
\psi= & \frac{\alpha}{1+\alpha} V_{0} \frac{1}{1+\frac{\sqrt{\sigma_{L} \sigma_{T}}}{\sigma_{b}}+\lambda k \frac{\sigma_{i}^{l}}{\sigma_{e}^{l}} \sqrt{\frac{\sigma_{L}}{\sigma_{T}}}} \sin k x \\
& \times\left[e^{\delta k z}+\lambda k \frac{\sigma_{i}^{t}-\alpha \sigma_{e}^{t}}{(1+\alpha) \sigma_{e}^{t}} \sqrt{\frac{\sigma_{L}}{\sigma_{T}}} e^{z / \lambda}\right]
\end{aligned}
$$

As the product $\lambda k$ tends to zero, the solution (44) tends to the solution obtained by Patel and Roth [4].

In order to quantify the difference between the solution (44) and the solution of Patel and Roth (which will be labelled $\left.\psi_{P R}\right)$, consider a comparison of the coefficient of $\sin k x$ at the surface $z=0$ as a function of $k$. Using the set of parameters given in [4]: $\sigma_{i}^{l}=0.2 \mathrm{~S} / \mathrm{m}, \sigma_{e}^{l}=0.2 \mathrm{~S} / \mathrm{m}, \sigma_{i}^{t}=0.02 \mathrm{~S} / \mathrm{m}$, $\sigma_{e}^{t}=0.08 \mathrm{~S} / \mathrm{m}$ and $\sigma_{b}=1.0 \mathrm{~S} / \mathrm{m}$ and defining the relative error as $\left|\left(\psi-\psi_{P R}\right) / \psi_{P R}\right|$, it can be shown via a simple asymptotic argument for small values of $\lambda k$ that the relative error is given by approximately $\frac{5}{3} \lambda k$. So, for example, if $\lambda k=0.01$, then the relative error is of the order of 0.0167 .

The above argument shows that, for this example, solving the equations with a method that makes fewer approximations does not significantly improve the accuracy of the solutions obtained by Patel and Roth [4]. The argument does, however, quantify the effects of the approximations made by Patel and Roth.

\section{EXAMPLE 3: PLANAR SLAB MODEL}

Consider a slab of cardiac tissue of thickness $2 a$ with parallel fibres aligned along the $z$-direction. An action potential propagates in the $z$-direction, which is independent of $x$, with the $y$-direction being parallel to the tissue surface (Figure 4). The tissue is perfused by a bath for $|y|>a$, and so the problem is symmetric about the plane $y=0$. The governing equations are (5) and (7), which can be written for this example as

$$
\sigma_{T} \frac{\partial^{2} \psi}{\partial y^{2}}+\sigma_{L} \frac{\partial^{2} \psi}{\partial z^{2}}=0
$$

and

$$
\frac{\partial^{2} \phi_{b}}{\partial y^{2}}+\frac{\partial^{2} \phi_{b}}{\partial z^{2}}=0
$$

In terms of the original variables $\phi_{i}$ and $\phi_{e}$, the boundary conditions necessary to solve this problem are given by (8), (9) and (10) at $y=a$, along with at $y=0$

$$
\frac{\partial \phi_{i}}{\partial y}=\frac{\partial \phi_{e}}{\partial y}=0
$$

This last condition implies that at $y=0$

$$
\frac{\partial \phi_{m}}{\partial y}=\frac{\partial \psi}{\partial y}=0
$$

To solve this problem Patel and Roth [4] write

$$
\phi_{m}=\phi_{m}^{0}+A e^{-(y-a) / \lambda}
$$

and

$$
\psi=\psi^{0}+B e^{-(y-a) / \lambda}
$$

using the exponential function as the auxiliary function. However, this choice of $\phi_{m}$ and $\psi$ violates the boundary condition (48), since $\phi_{m}^{0}$ is assumed to be a planar wave front with no variation perpendicular to the surface.

In order to overcome this problem, it is more correct to choose an auxiliary function based on the hyperbolic cosine, writing

$$
\phi_{m}=\phi_{m}^{0}+A \frac{\cosh (y / \lambda)}{\cosh (a / \lambda)}
$$

and

$$
\psi=\psi^{0}+B \frac{\cosh (y / \lambda)}{\cosh (a / \lambda)}
$$

This choice of the auxiliary function takes on the value 1 at the bath-tissue interface at $y=a$ and ensures that the boundary conditions (47) are satisfied.

To find a relationship between $A$ and $B$, substitute (49) and (50) into (5) and consider only derivatives perpendicular to the surface. After some algebra, this yields

$$
B=-\frac{1}{1+\alpha} \frac{\sigma_{i}^{t}-\alpha \sigma_{e}^{t}}{\sigma_{i}^{t}+\sigma_{e}^{t}} A
$$

as obtained by Patel and Roth [4]. Next, using the boundary condition (13) gives

$$
A=-\lambda \frac{\partial \psi_{0}}{\partial y} \frac{\sigma_{i}^{t}+\sigma_{e}^{t}}{\sigma_{e}^{t}} \operatorname{coth}(a / \lambda)
$$


which leads to

$$
B=\lambda \frac{\partial \psi_{0}}{\partial y} \frac{\sigma_{i}^{t}-\alpha \sigma_{e}^{t}}{\sigma_{e}^{t}(1+\alpha)} \operatorname{coth}(a / \lambda)
$$

Finally, substituting (52) and (53) into the boundary conditions (11) and (12), the following boundary conditions at $y=a$ for $\psi_{0}$ and $\phi_{b}$ are obtained

$$
\phi_{b}=\psi_{0}-\frac{\alpha}{1+\alpha} \phi_{m}^{0}+\lambda \frac{\partial \psi_{0}}{\partial y} \frac{\sigma_{i}^{t}}{\sigma_{e}^{t}} \operatorname{coth}(a / \lambda)
$$

and

$$
\sigma_{T} \frac{\partial \psi_{0}}{\partial y}=\sigma_{b} \frac{\partial \phi_{b}}{\partial y}
$$

where the assumption that $\lambda$ is small compared to the spatial scale has been ignored. The boundary condition (55) is identical to that obtained by Patel and Roth [4], but (54) is a new mixed boundary condition. Clearly letting $\lambda \rightarrow 0$ results in the boundary condition obtained by Patel and Roth.

The task now is to solve the equations (45) and (46). Substituting the approximations (49) and (50) into (45) and (46) gives

$$
\sigma_{T} \frac{\partial^{2} \psi_{0}}{\partial y^{2}}+\sigma_{L} \frac{\partial^{2} \psi_{0}}{\partial z^{2}}=0
$$

and

$$
\frac{\partial^{2} \phi_{b}}{\partial y^{2}}+\frac{\partial^{2} \phi_{b}}{\partial z^{2}}=0
$$

These two equations are solved subject to the boundary conditions (12) and (55). As discussed by Patel and Roth [4], the equations are best solved in Fourier transform space and yield

$$
\widehat{\psi_{0}}(y, k)=\frac{\alpha}{1+\alpha} \frac{\cosh (\delta k y)}{\beta(|k|, a, \delta, \lambda) \cosh (\delta k a)} \widehat{\phi_{m}^{0}}(k)
$$

and

$$
\widehat{\phi_{b}}(y, k)=-\frac{\alpha}{1+\alpha} \frac{\sigma_{T}}{\sigma_{b}} \delta \frac{e^{-k(y-a)}}{\beta(|k|, a, \delta, \lambda)} \tanh (\delta k a) \widehat{\phi_{m}^{0}}(k)
$$

where

$$
\widehat{\phi_{m}^{0}}(k)=\int_{-\infty}^{\infty} \phi_{m}^{0}(z) e^{i k z} d z
$$

and

$\beta(|k|, a, \delta, \lambda)=1+\frac{\sigma_{T}}{\sigma_{b}} \delta \tanh (\delta k a)+\lambda \delta k \tanh (\delta k a) \operatorname{coth} a / \lambda$.

Substituting equations (58) and (53) into a transformed version of equation (50) gives

$$
\begin{aligned}
\widehat{\psi}(y, k)= & \frac{\alpha}{1+\alpha} \frac{1}{\beta(|k|, a, \delta, \lambda)} \frac{\cosh (\delta k y)}{\cosh (\delta k a)} \\
& \times\left[1+\lambda \delta k \tanh (\delta k y) \frac{\cosh (y / \lambda)}{\sinh (a / \lambda)} \frac{\sigma_{i}^{t}-\alpha \sigma_{e}^{t}}{(1+\alpha) \sigma_{e}^{t}}\right] .
\end{aligned}
$$



FIG. 5: Relative error between surface potentials for two approximate solutions for propagation in a slab of cardiac tissue of finite thickness.

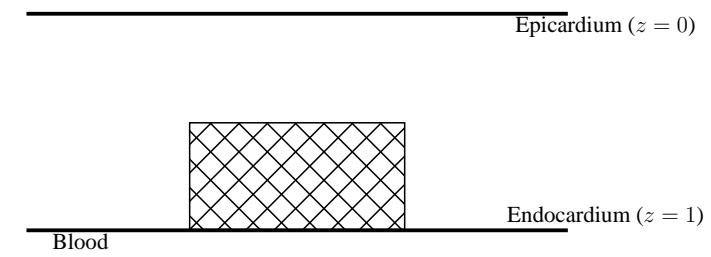

FIG. 6: A slab of cardiac tissue, insulated on the epicardium (at $z=$ 0 ) and in contact with a blood mass on the endocardium (at $z=1$ ). The hatched region represents a region of subendocardial ischaemia.

Taking the limit as $\lambda \rightarrow 0$ gives the same solution as obtained previously [4].

To contrast the difference between the solution obtained above (60) and that obtained by Patel and Roth [4], consider the relative error as defined above, but in terms of transformed functions. Figure 5 shows the relative error on the bath-tissue interface, $y=a$, against frequency, $k$, for values of $a$ of $1.5 \mathrm{~mm}$ (typical of the thickness of the atrial wall) and $5 \mathrm{~mm}$ (typical of the thickness of the ventricular wall) using the same conductivity values as above. The figure shows that the errors increase as $k$ increases, but for lower frequencies they are generally less than 0.005 . It is interesting to note that the errors are larger for the ventricular wall $(a=5 \mathrm{~mm})$ than for the atrial wall ( $a=1.5 \mathrm{~mm})$. Again, as in the previous example, it appears that the extra effort expended in using solutions that satisfy the boundary conditions exactly is not justified.

\section{EXAMPLE 4: MODELLING SUBENDOCARDIAL ISCHAEMIA DURING THE ST SEGMENT}

Consider a slab of cardiac tissue, infinite in the $x$ and $y$ directions, insulated by the $x y$ plane at $z=0$ and in contact with an infinite blood mass at $z=1 \mathrm{~cm}$ (Figure 6). It can be shown that the governing equation for the extracellular potential $\phi_{e}$ in the tissue is given by

$$
\nabla \cdot\left(\mathbf{M}_{i}+\mathbf{M}_{e}\right) \nabla \phi_{e}=-\nabla \cdot \mathbf{M}_{i} \nabla \phi_{m}
$$


with $\phi_{m}=\phi_{i}-\phi_{e}$. The potential in the blood (bath) satisfies Laplace's equation

$$
\nabla^{2} \phi_{b}=0
$$

The boundary conditions required to solve the equations are usually that $\phi_{e} \rightarrow 0$ and $\phi_{b} \rightarrow 0$ as $|x| \rightarrow \infty$ and $|y| \rightarrow \infty$ and that $\phi_{b} \rightarrow 0$ as $z \rightarrow \infty$. It is also required that

$$
\begin{aligned}
& \text { at } z=0, \frac{\partial \phi_{e}}{\partial z}=0 \text { and } \frac{\partial \phi_{i}}{\partial z}=0 \\
& \text { at } z=1, \phi_{e}=\phi_{b} \text { and } \sigma_{e}^{t} \frac{\partial \phi_{e}}{\partial z}=\sigma_{b} \frac{\partial \phi_{b}}{\partial z} .
\end{aligned}
$$

(See [6] for complete details of the model.)

In general, to solve the problem, $\phi_{m}$ is prescribed and depends on the size and position of the ischaemic region in the tissue. Also, it is assumed that

$$
\left.\frac{\partial \phi_{m}}{\partial z}\right|_{z=0}=0,\left.\frac{\partial \phi_{m}}{\partial z}\right|_{z=1}=0 \text { and }\left.\phi_{m}\right|_{z=1}=-\Delta \phi
$$

where $\Delta \phi$ is the difference in ST potentials between the normal and ischaemic regions [9]. These conditions imply that the condition

$$
\left.\frac{\partial \phi_{i}}{\partial z}\right|_{z=1}=0
$$

cannot be satisfied exactly. However, it is generally argued that if the length constant of the tissue in the transverse direction is small compared to the depth of the tissue, then condition (64) represents an acceptable approximation [6-8].

Following the ideas of Patel and Roth [4], the definitions

$$
\phi_{m}=\phi_{m}^{0}+A e^{-(1-z) / \lambda}
$$

and

$$
\phi_{e}=\phi_{e}^{0}+B e^{-(1-z) / \lambda}
$$

make it possible to satisfy the boundary condition (65). However, this choice for the functional representations of $\phi_{m}$ and $\phi_{e}$ violates the boundary condition $\left.\frac{\partial \phi_{i}}{\partial z}\right|_{z=0}=0$.

More appropriate definitions for $\phi_{m}$ and $\phi_{e}$ should again use an auxiliary function based on the hyperbolic cosine function; that is, write

$$
\phi_{m}=\phi_{m}^{0}+A \frac{\cosh (z / \lambda)}{\cosh (1 / \lambda)}
$$

and

$$
\phi_{e}=\phi_{e}^{0}+B \frac{\cosh (z / \lambda)}{\cosh (1 / \lambda)}
$$

where $\phi_{m}^{0}$ is a function which captures the general shape of the transmembrane potential distribution, in particular that $\left.\frac{\partial \phi_{m}^{0}}{\partial z}\right|_{z=1}=0$.
Substituting (68) and (69) into equation (61) and requiring that $\left.\frac{\partial \phi_{i}}{\partial z}\right|_{z=1}=0$ gives

$$
A=-\left.\lambda \operatorname{coth}(1 / \lambda) \frac{\sigma_{i}^{t}+\sigma_{e}^{t}}{\sigma_{e}^{t}} \frac{\partial \phi_{e}^{0}}{\partial z}\right|_{z=1}
$$

and

$$
B=\left.\lambda \operatorname{coth}(1 / \lambda) \frac{\sigma_{i}^{t}}{\sigma_{e}^{t}} \frac{\partial \phi_{e}^{0}}{\partial z}\right|_{z=1}
$$

where $\phi_{e}^{0}$ satisfies

$$
\nabla \cdot\left(\mathbf{M}_{i}+\mathbf{M}_{e}\right) \nabla \phi_{e}^{0}=-\nabla \cdot \mathbf{M}_{i} \nabla \phi_{m}^{0}
$$

subject to

$$
\begin{aligned}
\text { at } z & =0, \quad \frac{\partial \phi_{e}^{0}}{\partial z}=0 \\
\text { at } z & =1, \quad \phi_{b}=\phi_{e}^{0}+\lambda \operatorname{coth}(1 / \lambda) \frac{\sigma_{i}^{t}}{\sigma_{e}^{t}} \frac{\partial \phi_{e}^{0}}{\partial z} \\
\text { and at } z & =1, \quad \sigma_{b} \frac{\partial \phi_{b}}{\partial z}=\sigma_{e}^{t}\left(1+\frac{\sigma_{i}^{t}}{\sigma_{e}^{t}}\right) \frac{\partial \phi_{e}^{0}}{\partial z}
\end{aligned}
$$

with $\phi_{b}$ satisfying (62) such that $\phi_{b} \rightarrow 0$ as $z \rightarrow \infty$. The same boundary conditions apply to $\phi_{e}^{0}$ and $\phi_{b}$ in the $x$ and $y$ directions as described above.

The problem in terms of $\phi_{e}^{0}, \phi_{m}^{0}$ and $\phi_{b}$ is exactly that described previously [6] and can be solved in an identical manner. There is, however, a slight change to one entry in the coefficient matrix in the one dimensional numerical scheme used in [6], due to the new mixed boundary condition at $z=1$, equation (72). The extracellular potential, $\phi_{e}$ can be obtained by calculating $\phi_{e}^{0}$ via the methods described in [6] and using

$$
\phi_{e}=\phi_{e}^{0}+\left.\lambda \operatorname{coth}(1 / \lambda) \frac{\sigma_{i}^{t}}{\sigma_{e}^{t}} \frac{\partial \phi_{e}^{0}}{\partial z}\right|_{z=1} \frac{\cosh (z / \lambda)}{\cosh (1 / \lambda)}
$$

Figure 7 shows the epicardial $(z=0)$ surface potential distributions, for a $16 \mathrm{~cm} \times 16 \mathrm{~cm}$ slab of cardiac tissue, $1 \mathrm{~cm}$ thick containing a $4 \mathrm{~cm} \times 4 \mathrm{~cm}$ region of $50 \%$ ischaemia, obtained using (a) the method described in [6] and (b) using the modified methods described here (equation (74)). The conductivity values used are those given by Clerc [21] and the slab contains no fibre rotation. The main difference between the two figures is the shape of the contour at $-0.6 \mathrm{mV}$. For the solution method described in [6], this particular contour is a single continuous curve (Figure 7(a)), while the modifications introduced here produce three distinct curves (Figure 7(b)). Also, equation (74) indicates a slightly higher minimum potential and a slightly higher maximum potential. Interestingly, the solution obtained from equation (74) more closely matches a numerical solution for the same problem based on the finite volume method [11].

As a second example, consider the same slab of cardiac tissue, this time with $120^{\circ}$ fibre rotation from the epicardium to the endocardium with the fibres oriented along the $x$-axis on the epicardium. The epicardial potential distributions are 
(a)

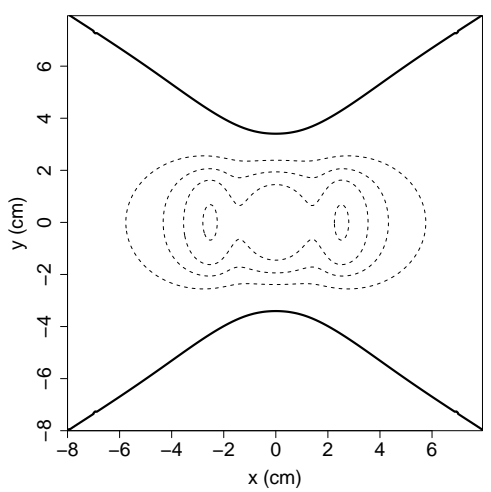

(b)



FIG. 7: Epicardial potential distributions on the surface of a slab of cardiac tissue with no fibre rotation. Negative potentials are indicated by the dashed lines and the zero of potential is denoted by the thick solid line. The contour interval is $0.2 \mathrm{mV}$. Panel (a) shows the solution obtained from [6] (with a minimum potential of $-0.8279 \mathrm{mV}$ and maximum potential of $0.0276 \mathrm{mV}$ ) and panel (b) is the solution obtained using the methods described here (with a minimum potential of $-0.8205 \mathrm{mV}$ and maximum potential of $0.0292 \mathrm{mV}$ ).

shown in Figure 8 with panel (a) showing the distribution obtained using the techniques from [6] and panel (b) showing the distribution from equation (74). In this example the differences between the two contour plots are very difficult to see with the naked eye. The main distinctions between the two plots are the slight differences between the extremes in the potential values.

Similar results can be achieved when considering the cylindrical model of the left ventricle [8]. In that case it would be necessary to choose

$$
\phi_{m}=\phi_{m}^{0}+A \frac{I_{0}((b-r) / \lambda)}{I_{0}((b-a) / \lambda)}
$$

with a similar expression for $\phi_{e}$. Here the auxiliary function is based on $I_{0}$, the zero order modified Bessel function with $a$ and $b$ representing the inner and outer radii of the ventricular muscle, respectively.

It was mentioned by Patel and Roth [4] that there is a difference in the coefficients obtained by ignoring and then allowing for satisfaction of the boundary condition (10). Using their (a)



(b)

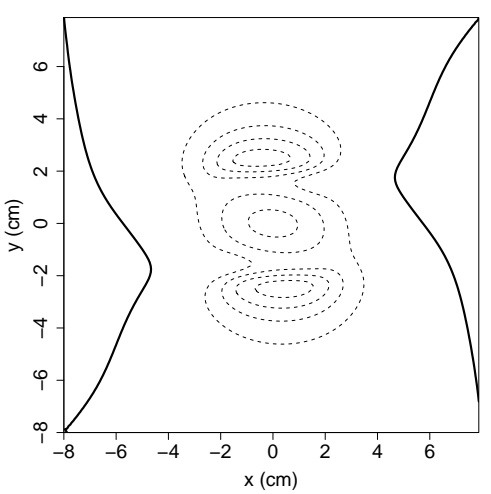

FIG. 8: Epicardial potential distributions on the surface of a slab of cardiac tissue with $120^{\circ}$ of fibre rotation. Negative potentials are indicated by the dashed lines and the zero of potential is denoted by the thick solid line. The contour interval is $0.2 \mathrm{mV}$. Panel (a) is the solution obtained from [6] (with a minimum potential of $-0.9149 \mathrm{mV}$ and maximum potential of $0.0099 \mathrm{mV}$ ) and panel (b) is the solution obtained using the methods described here (with a minimum potential of $-0.9084 \mathrm{mV}$ and maximum potential of $0.0113 \mathrm{mV}$ ).

conductivity data, there is a difference of $17 \%$ [4] in the coefficient relating to the bath potential, but there is a difference of only $3 \%$ in the coefficient relating to the tissue potential. They claim that the differences may be significant in precise quantitative measurements, but are probably not important for qualitative analysis of the ECG. Figures 7 and 8 show the extent of this difference for the case of subendocardial ischaemia during the ST segment. There may be more significant differences in studies of other aspects of the ECG and perhaps the differences are manifest more strongly in the bath than in the tissue.

\section{CONCLUSIONS}

This paper has presented an analysis of the approximate methods suggested by Patel and Roth $[4,5]$ for solving problems in electrocardiography. It has been shown that their approximation method can be improved for defibrillation prob- 
lems by considering auxiliary functions which are more appropriate to the geometry of the problem under consideration. In the example considered here, using a modified Bessel function as the auxiliary function gave more accurate solutions to the defibrillation of a thin fibre than using an exponential function [5].

For electrocardiogram problems, it was shown that choosing auxiliary functions that satisfied all boundary conditions and ensuring that all boundary conditions were satisfied during the solution process provided solutions that were not significantly different to those produced by the original approximate methods [4]. This demonstrates the appropriateness of the original approximations introduced by Patel and Roth.

One interesting result from the analysis performed in this paper was that the approximate method of Patel and Roth [4] is equivalent to an approach published by Roth and Wikswo [19] some twenty years ago. Hence it can be concluded that for cardiac propagation problems, good approximate solutions to equations (1) and (2) subject to boundary conditions (8), (9) and (10) can be obtained by solving equations (1) and (2) subject to boundary conditions (8) and (33) and assuming that the transmembrane potential does not vary in the direction normal to the fibre direction.

Finally, for problems simulating epicardial potential distributions from subendocardial ischaemia during the ST segment, the approximate solutions obtained using the methods described here more closely reproduce the full numerical solution than those published previously [6].
[1] L. Tung, Ph.D. thesis, Massachusetts Institute of Technology (1978).

[2] I. J. LeGrice, B. H. Smaill, L. Z. Chai, S. G. Edgar, J. B. Gavin, and P. J. Hunter, Am. J. Physiol. 269, H571 (1995).

[3] O. H. Schmitt, in Information Processing in the Nervous System, edited by K. N. Leibovic (Springer-Verlag, New York, 1969), chap. 18 , pp. 325-331.

[4] S. G. Patel and B. J. Roth, Physical Review E (Statistical, Nonlinear, and Soft Matter Physics) 72, 051931 (pages 7) (2005), URL http: / / link. aps.org/abstract/PRE/ v72/e051931.

[5] S. G. Patel and B. J. Roth, Physical Review E 71 (2005).

[6] P. R. Johnston, D. Kilpatrick, and C. Y. Li, IEEE Transactions on Biomedical Engineering 48, 1366 (2001).

[7] P. R. Johnston and D. Kilpatrick, IEEE Transactions on Biomedical Engineering 50, 150 (2003).

[8] P. R. Johnston, Mathematical Biosciences 186, 43 (2003).

[9] P. R. Johnston, Mathematical Biosciences 198, 97 (2005).

[10] M. C. MacLachlan, J. Sundnes, O. Skavhaug, M. Lysaker, B. F. Nielsen, and A. Tveito, Mathematical Biosciences 210, 238 (2007), URL http://www.sciencedirect. com/science/article/B6VHX-4NKJ0PK-1/2/ 1 a 8 be2b7ee6181ead81060dea3a628d4.

[11] R. C. Penland, D. M. Harrild, and C. S. Henriquez, Computing and Visualization in Science 4, 215 (2002), URL http: // dx.doi.org/10.1007/s00791-002-0078-4.

[12] M. Trew, I. Le Grice, B. Smaill, and A. Pullan, Annals of Biomedical Engineering 33, 590 (2005), URL http: //www. springerlink. com/openurl.asp?genre= article\&id=doi:10.1007/s10439-005-1434-6.

[13] J. Sundnes, G. T. Lines, and A. Tveito, Mathematical Biosciences 194, 233 (2005).

[14] B. M. Johnston, P. R. Johnston, and D. Kilpatrick, Mathematical Biosciences 202, 288 (2006).

[15] B. M. Johnston, P. R. Johnston, and D. Kilpatrick, Computer Methods in Biomechanics and Biomedical Engineering 11, 223 (2008), URL http: //www. informaworld. com/10.1080/10255840701747594.

[16] B. J. Roth, IEEE Transactions on Biomedical Engineering 44, 326 (1997).

[17] B. J. Roth and D. L. Beaudoin, Physical Review E 67 (2003).

[18] J. C. Clements and B. M. Horáček, IEEE Transactions on Biomedical Engineering 52, 1784 (2005).

[19] B. J. Roth and J. P. Wikswo, Jr., IEEE Transactions on Biomedical Engineering BME-33, 467 (1986).

[20] W. Krassowska and J. Neu, IEEE Transactions on Biomedial Engineering 41, 143 (1994).

[21] L. Clerc, Journal of Physiology 255, 335 (1976). 